\title{
Multivariate Analysis Applications to Crystallography
}

\author{
Rocco Caliandro ${ }^{1, *(1)}$ and Marco Milanesio ${ }^{2, *(D)}$ \\ 1 Institute of Crystallography, CNR, via Amendola, 122/o, 70126 Bari, Italy \\ 2 Dipartimento di Scienze e Innovazione Tecnologica, Università del Piemonte Orientale, viale T. Michel 11, \\ 15121 Alessandria, Italy \\ * Correspondence: rocco.caliandro@ic.cnr.it (R.C.); marco.milanesio@uniupo.it (M.M.)
}

Citation: Caliandro, R.; Milanesio, M. Multivariate Analysis Applications to Crystallography. Crystals 2021, 11, 166. https://doi.org/10.3390/ cryst11020166

Received: 4 February 2021 Accepted: 5 February 2021 Published: 8 February 2021

Publisher's Note: MDPI stays neutral with regard to jurisdictional claims in published maps and institutional affiliations.

Copyright: (C) 2021 by the authors. Licensee MDPI, Basel, Switzerland. This article is an open access article distributed under the terms and conditions of the Creative Commons Attribution (CC BY) license (https:// creativecommons.org/licenses/by/ $4.0 /)$.
The Special Issue contributions cover the main themes related to the applications of multivariate analysis to crystallography. Seven manuscripts in the fields of chemistry, physics, and earth and materials science were published. The power of factor analysis when applied to the classification of rocks was demonstrated in the two papers by Fomina et al. and Kozlov et al., the first [1] dealing with the algorithm used for data analysis and the second [2] with the geological interpretation of the data. The significance and theoretical development behind the Principal Component Analysis (PCA) applied to X-ray powder diffraction were then investigated by Chernyshov et al. [3]. The authors, starting from previous works on the topic, further developed guidelines for the interpretation of PCA results, aiming to facilitate data analysis and correctly extract the structural information. PCA potentialities in analyzing in situ X-ray powder diffraction (XRPD) data were elucidated by Conterosito et al. [4]. Here, the authors demonstrated how PCA can support traditional XRPD data refinement methods to obtain the best possible location of gas molecules within a Y zeolite. In the paper by Ishihara et al. [5], multivariate analysis demonstrated its power when the probe is completely different from traditional diffraction techniques: a cocrystal formation reaction was followed by teraHertz spectroscopy, and the dataset was successfully analyzed using the multivariate curve resolution approach.

Finally, two reviews analyzed the state of the art in the two main X-ray-based approaches in elucidating short- and long-range ordered structures. Martini and Borfecchia [6] summarized the advances in the use of multivariate analysis for the investigation of the local structure by X-ray adsorption techniques. Guccione et al. [7] collected and categorized the possible multivariate approaches for the analysis of structure ray diffraction data from single crystal and powder crystalline samples. In both cases, multivariate analysis resulted in a promising complementary tool to the traditional approach, which is able to speed up and/or increase the amount of useful information extracted for the data. Huge developments and widespread applications are envisaged, especially in all cases where new sources and detectors allow the collection of giga- to tera-bite data in in situ or operando experiments, carried out in laboratory or large-scale facilities, such as synchrotrons and X-ray Free Electron Lasers (XFELs). This Special Issue can thus be a useful guide for the many scientists interested in applying multivariate analysis in crystallography, both from theoretical and practical viewpoints.

Conflicts of Interest: The authors declare no conflict of interest.

\section{References}

1. Fomina, E.; Kozlov, E.; Bazai, A. Factor Analysis of XRF and XRPD Data on the Example of the Rocks of the Kontozero Carbon-atite Complex (NW Russia). Part I: Algorithm. Crystals 2020, 10, 874. [CrossRef]

2. Kozlov, E.; Fomina, E.; Khvorov, P. Factor Analysis of XRF and XRPD Data on the Example of the Rocks of the Kontozero Car-bonatite Complex (NW Russia). Part II: Geological Interpretation. Crystals 2020, 10, 873. [CrossRef]

3. Chernyshov, D.; Dovgaliuk, I.; Dyadkin, V.; van Beek, W. Principal Component Analysis (PCA) for Powder Diffraction Data: Towards Unblinded Applications. Crystals 2020, 10, 581. [CrossRef] 
4. Conterosito, E.; Lopresti, M.; Palin, L. In Situ X-ray Diffraction Study of Xe and CO2 Adsorption in Y Zeolite: Comparison between Rietveld and PCA-Based Analysis. Crystals 2020, 10, 483. [CrossRef]

5. Ishihara, S.; Hattori, Y.; Otsuka, M.; Sasaki, T. Cocrystal Formation through Solid-State Reaction between Ibuprofen and Nico-tinamide Revealed Using THz and IR Spectroscopy with Multivariate Analysis. Crystals 2020, 10, 760. [CrossRef]

6. Martini, A.; Borfecchia, E. Spectral Decomposition of X-ray Absorption Spectroscopy Datasets: Methods and Applications. Crystals 2020, 10, 664. [CrossRef]

7. Guccione, P.; Lopresti, M.; Milanesio, M.; Caliandro, R. Multivariate Analysis Applications in X-ray Diffraction. Crystals 2021, 11, 12. [CrossRef] 